\title{
Optoelectrical properties of four amorphous silicon thin-film transistors 200 dpi active-matrix organic polymer light-emitting display
}

\author{
Yongtaek Hong, Jeong-Yeop Nahm, ${ }^{\text {a) }}$ and Jerzy Kanicki ${ }^{\text {b) }}$ \\ Solid-State Electronics Laboratory, Department of EECS, University of Michigan, Ann Arbor, Michigan 48105
}

(Received 3 April 2003; accepted 16 August 2003)

\begin{abstract}
We report on opto-electrical properties of a current-driven 200 dpi active-matrix organic polymer red light-emitting display (AM-PLED) based on four hydrogenated amorphous silicon thin-film transistor pixel electrode circuits. The AM-PLED luminance and effective light-emission efficiency were $30 \mathrm{~cd} / \mathrm{m}^{2}$ and $0.3 \mathrm{~cd} / \mathrm{A}$, respectively, at the data current equal to $25 \mathrm{~mA}$. The display electroluminescent spectrum has a peak located at and the full width at half maximum value of 644 and $95 \mathrm{~nm}$, respectively, and Commission Internationale de l'Eclairage color coordinates of (0.66,0.33). (C) 2003 American Institute of Physics. [DOI: 10.1063/1.1617372]
\end{abstract}

Since hydrogenated amorphous silicon $(a-\mathrm{Si}: \mathrm{H})$ thinfilm transistors (TFTs) active-matrix (AM) arrays can be fabricated at low cost and with a high uniformity over large areas, it is expected that one day this mature technology could become preferred in comparison with the polycrystalline silicon (poly-Si) TFTs technology ${ }^{1-3}$ for the activematrix organic light-emitting displays (AM-OLEDs). Furthermore, recent enhancements of the organic light-emitting device (OLED) performances ${ }^{4}$ have made it easier to extend the $a$-Si:H TFTs technology to AM-OLED. In AM-OLED, $a-\mathrm{Si}: \mathrm{H}$ TFTs act as switching and driving devices in pixel electrode circuits. To drive light-emitting devices in AMOLEDs, a continuous excitation during the whole frame period is needed. This can be a very demanding task for $a-\mathrm{Si}: \mathrm{H}$ TFTs which can produce undesirable variations in their electrical properties. To compensate for these variations current driven pixel electrode circuits have been proposed for AM-OLEDs. ${ }^{1,5,6}$

In this letter, we report on optoelectronic properties of a current-driven 4- $a$-Si:H TFTs AM-organic polymer lightemitting display (AM-PLED). Its properties are compared to typical characteristics of organic polymer light-emitting devices (PLEDs).

The fabrication of 4-a-Si:H TFTs 200 dpi active-matrix arrays consists of six masks process steps: chromium (2000 $\AA$ ) gates and selection lines are defined on Coming 1737 glass substrates (mask No. 1); $a-\mathrm{SiN} x: \mathrm{H}(3000 \AA) / a-\mathrm{Si}: \mathrm{H}$ (1000 $\AA) / n^{+} a$-Si:H (300 $\AA$ ) trilayer is deposited by the plasma-enhanced chemical vapor deposition (PECVD) method, then device active islands are defined (mask No. 2); gate electrode via through gate insulator is formed (mask No. 3); molybdenum (2000 ̊) source/drain and data lines are defined (mask No. 4); backchannel etching and thermal annealing are performed; benzocyclobutene (BCB) $(1-1.5 \mu \mathrm{m})$ planarization layer is deposited and thermally cured; drain electrode via through the BCB layer is defined (mask No. 5); indium tin oxide (ITO) (1000 $\AA$ ) is deposited and pixel electrodes are defined over the BCB layer (mask No. 6). The ITO

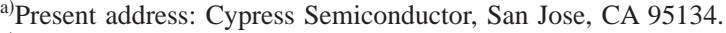

b) Author to whom correspondence should be addressed; electronic mail: kanicki@eecs.umich.edu
}

surface is UV-ozone treated for $10 \mathrm{~min}$ before a hole injection layer [poly $(3,4$-ethylene dioxythiophene) doped with poly (styrenesulfonate)] is deposited from a water solution by spin coating and is thermally cured. Next the red lightemissive poly-fluorene layer is deposited from solution by spin coating and is thermally cured. Finally a calcium (150 $\AA$ )/aluminum (2000 ̊) bilayer cathode is thermally evaporated on top of the display. We removed the PLED active layers from display contact pads using organic solvents. A schematic cross section of the PLED is shown in the inset of Fig. 2. All the cathode electrodes for each pixel are connected in the AM-PLED. To compare the optoelectrical properties of the AM-PLED with those of typical PLEDs, we also fabricated a red light-emitting PLED $\left(2 \times 3 \mathrm{~mm}^{2}\right)$ over the ITO-coated glass substrate, which has the same organic active layer structure as the AM-PLED.

To evaluate the AM-PLED properties we applied con-

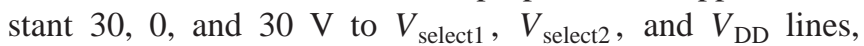
respectively. ${ }^{6}$ The data current ranging from 0 to $25 \mathrm{~mA}$ was applied to $I_{\text {data }}$ lines to light up the display, and the display luminance was measured for each data current level. The light was emitted through the ITO electrode. Figure 1 shows an image of the red light-emitting 200 dpi AM-PLED for data current of $25 \mathrm{~mA}$; the magnified image of the lightemitting pixels is also included in this figure. The fabrication yield of the AM-PLED light-emitting pixels was about $75 \%$, the display size was $0.5 \times 0.5$ in. $^{2}(100 \times 100$ pixels $)$ and the pixel aperture ratio (AR) was about $10 \%$. The AR was defined as the ratio of the pixel PLED area $\left(24 \times 65 \mu \mathrm{m}^{2}\right)$ to the whole pixel area $\left(127 \times 127 \mu \mathrm{m}^{2}\right)$.

The total luminous flux of the AM-PLED and PLEDs have been measured in air at room temperature, using an integrating sphere and a calibrated photodetector connected to a radiometer. ${ }^{7}$ Figure 2 shows characteristic variation of the display luminous flux versus applied data current. We obtained up to $1.1 \times 10^{-2}$ lumen when the data current was equal to $25 \mathrm{~mA}$. For a Lambertian emitter, we can calculate the luminance $(L)$ from the measured luminous flux $(\Phi)$ :

$$
L=\frac{\Phi}{\pi \times A},
$$

where $A$ is the area of the light emitter. By assuming that the 


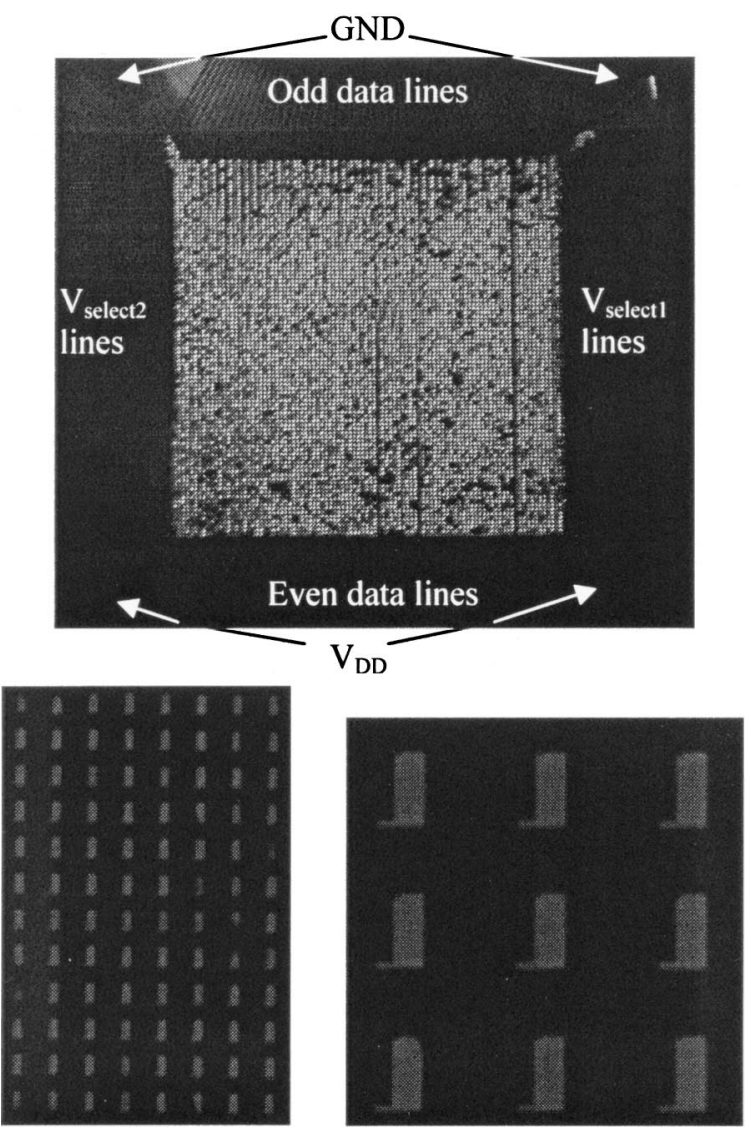

FIG. 1. Top view of illuminated 4- $a$-Si:H TFTs 200 dpi AM-PLED. Magnified images of the light-emitting pixels are also shown.

AM-PLED is a Lambertian emitter (which was verified experimentally), we calculated the display luminance $\left(L_{\text {Display }}\right)$ by considering the display area and the fabrication yield of the light-emitting pixels: $A=1.27 \mathrm{~cm} \times 1.27 \mathrm{~cm} \times 0.75$ $=1.215 \times 10^{-4} \mathrm{~m}^{2}$. Evolution of $L_{\text {Display }}$ with the data current is shown in Fig. 2; and for data current of $25 \mathrm{~mA}$ we obtained $L_{\text {Display }}=30 \mathrm{~cd} / \mathrm{m}^{2}$. In addition, if we take into consideration the pixel PLED area, e.g., pixel $\mathrm{AR}=10 \%$, we can calculate the effective light-emission luminance $\left(L_{\text {Emission }}\right)$ for $A=1.27 \mathrm{~cm} \times 1.27 \mathrm{~cm} \times 0.75 \times 0.1=1.215$ $\times 10^{-5} \mathrm{~m}^{2}$. The variation of $L_{\text {Emission }}$ versus effective current density (defined as data current/total effective currentflowing area of the AM-PLED) is shown in Fig. 3; and

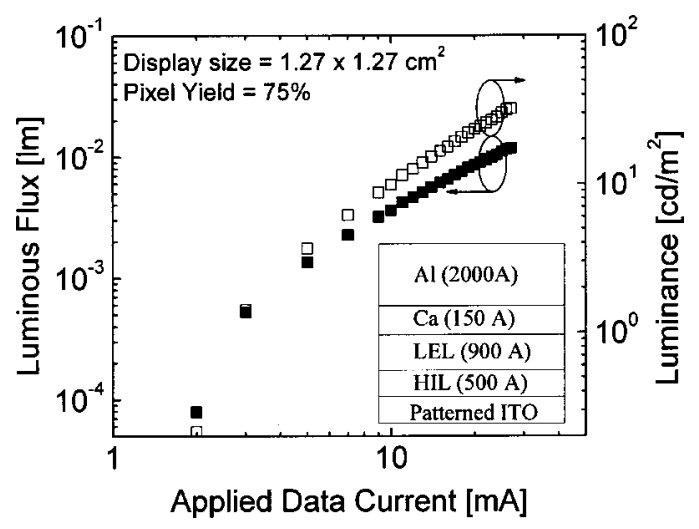

FIG. 2. The variations of luminous flux and luminance $\left(L_{\text {display }}\right)$ vs applied data current of 4- $a$-Si:H TFTs 200 dpi AM-PLED are shown. The structure of the organic polymer light-emitting device is also included in this figure.

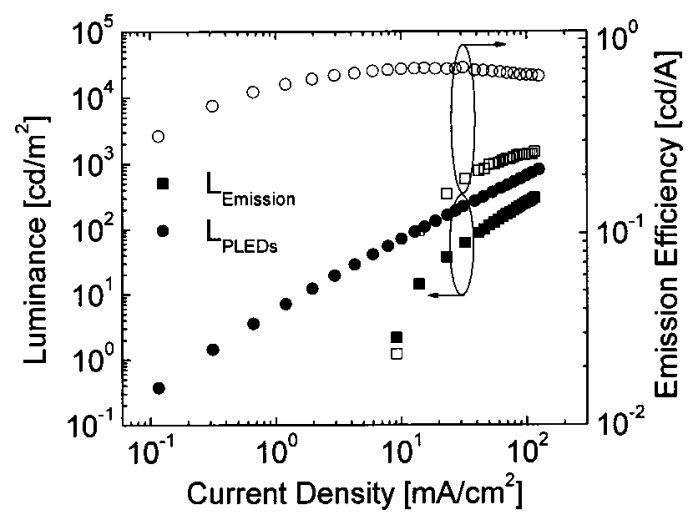

FIG. 3. The variations of effective light-emission luminance (solid square, $L_{\text {emission }}$ ) and effective light-emission efficiency (open square) vs effective current density of 4- $a$-Si:H TFTs 200 dpi AM-PLED are shown. The evolution of luminance (solid circle, $L_{\mathrm{PLED}}$ ) and light-emission efficiency (open circle) vs effective current density of the red PLED are also shown.

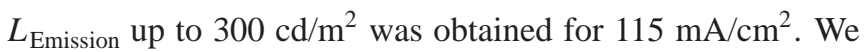
have also defined the effective light-emission efficiency of the AM-PLED as the ratio of effective light-emission luminance to effective current density. Its variation with the current density is also shown in Fig. 3; and for the studied displays, we obtained a maximum effective light-emission efficiency of about $0.3 \mathrm{~cd} / \mathrm{A}$ at $115 \mathrm{~mA} / \mathrm{cm}^{2}$. In Fig. 3, the luminance and light-emission efficiency of the red PLEDs $\left(2 \times 3 \mathrm{~mm}^{2}\right)$ are also shown. The PLED had a luminance of about $720 \mathrm{~cd} / \mathrm{m}^{2}$ at $110 \mathrm{~mA} / \mathrm{cm}^{2}$ and a maximum light emission efficiency of about $0.71 \mathrm{~cd} / \mathrm{A}$ at $31 \mathrm{~mA} / \mathrm{cm}^{2} @ 220$ $\mathrm{cd} / \mathrm{m}^{2}$. As shown in Fig. 3, the effective light-emission efficiency of the AM-PLED is lower in comparison with the light-emission efficiency of the PLEDs by a factor of 3-4 for the current density ranging from 80 to $110 \mathrm{~mA} / \mathrm{cm}^{2}$. It is speculated that this difference is due to leakage current through defective AM-PLED pixels that do not contribute to light emission.

In Fig. 4, the electroluminescent (EL) spectra of the red light-emitting AM-PLED and PLEDs are shown. EL spectra were measured by mounting a charge coupled device based spectrometer on the detector port of the integrating sphere. ${ }^{7,8}$ The wavelength of the spectrometer was calibrated using a standard mercury lamp. From the EL spectra we extracted their peak positions located at 644 and $653 \mathrm{~nm}$, and their full

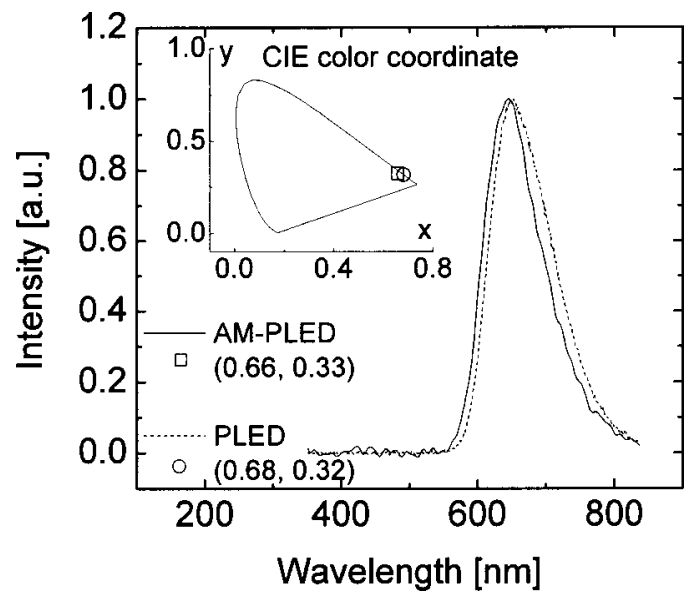

FIG. 4. EL spectra and CIE color coordinates of 4- $a$-Si:H TFTs 200 dpi AM-PLED (solid line) and PLED (dashed line) are shown. 
width at half maximum (FWHM) values of 95 and $105 \mathrm{~nm}$, for AM-PLED and PLEDs, respectively. From these spectra, we also calculated Commission Internationale de l'Eclairage (CIE) color coordinates for AM-PLED and PLEDs, which were $(0.66,0.33)$ and $(0.68,0.32)$, respectively, as shown in the inset of Fig. 4. The blueshift and smaller FWHM value of the AM-PLED EL spectrum is responsible for the decrease of CIE- $x$ and the increase of CIE- $y$ color coordinates, respectively.

In conclusion, we have fabricated and evaluated the optoelectrical properties of the current-driven $4-a-\mathrm{Si}: \mathrm{H}$ TFTs 200 dpi AM-PLED. The AM-PLED had luminescence of $30 \mathrm{~cd} / \mathrm{m}^{2}$ at $25 \mathrm{~mA}$ and its maximum effective light emission efficiency was about $0.3 \mathrm{~cd} / \mathrm{A}$ at $115 \mathrm{~mA} / \mathrm{cm}^{2}$. The AMPLED EL spectrum was blueshifted and narrower in comparison with the typical PLED EL spectrum. Overall the PLED showed better optical performance than the AMPLED.

This work was supported by a NIH grant. The authors would like to thank Dr. I. French at Philips Research Laboratory (UK) for assistance with the PECVD of different films. The authors also would like to thank Professor Reiji Hattori at Kyushu University (Japan) for many useful discus- sions related to pixel electrode circuits design and Dr. J.-H. Kim at University of Michigan (Ann Arbor) for technical assistance in the active-matrix arrays processing. The lightemission of 4- $a$-Si:H TFTs AM-PLED was observed on March 4, 2003.

${ }^{1}$ R. M. A. Dawson, S. Shen, D. A. Furst, S. Connor, J. Hsu, M. G. Kane, R. G. Stewart, A. Ipri, C. N. King, P. J. Green, R. T. Flegal, S. Pearson, W. A. Barrow, E. Dickey, K. Ping, S. Robinson, C. W. Tang, S. Van Slyke, F. Chen, J. Shi, M. H. Lu, M. Moskewicz, and J. C. Sturm, S.I.D. '99 Digest (Society for Information Display, San Jose, CA,), p. 438.

${ }^{2}$ T. Shimoda, M. Kimura, and S. Miyashita, Tech. Dig. Int. Electron Devices Meet. 99, 107 (1999).

${ }^{3}$ T. Sasaoka, M. Sekiya, A. Yumoto, J. Yamada, T. Hirano, Y. Iwase, T. Yamada, T. Ishibashi, T. Mori, M. Asano, S. Tamura, and T. Urabe, SID '01 Digest (Society for Information Display San Jose, CA, 2001), p. 384 .

${ }^{4}$ M. Hack, R. Kwong, M. S. Weaver, M. Lu, and J. J. Brown, Proceedings of the 2nd International Display Manufacturing Conference (2002), p. 57.

${ }^{5}$ Y. He, R. Hattori, and J. Kanicki, IEEE Electron Device Lett. 21, 590 (2000).

${ }^{6}$ J. Kanicki, J.-H. Kim, J.-Y. Nahm, Y. He, and R. Hattori, 21st International Display Conference, 16-19 October 2001 Nagoya, Japan (Society for Information Display, San Jose, CA, 2001), p. 315.

${ }^{7}$ Y. Hong and J. Kanicki, Rev. Sci. Instrum. 74, 3572 (2003).

${ }^{8}$ Y. Hong and J. Kanicki, 21st International Display Conference, 16-19 October 2001 Nagoya, Japan (Society for Information Display, San Jose, CA, 2001), p 1443. 